\title{
Utilização de metodologia ativa de ensino na formação do profissional de nutrição
}

The use of an active learning approach on the educational training of nutritionists

Utilización de metodología activa de enseñanza en la formación del profesional de nutrición

Cinthia Regina Sales Furtado Vieira ${ }^{1 *}$, Fábio Costa de Vasconcelos ${ }^{1}$, Jamilie Suelen dos Prazeres Campos$^{2}$, Rosa de Fátima da Silva Vieira Marques³, Marcia Bitar Portella ${ }^{3}$.

\section{RESUMO}

Objetivo: Avaliar a utilização das metodologias ativas como estratégia de ensino nos cursos de graduação em Nutrição de Belém-PA. Método: O estudo é descritivo transversal, quantitativo com aplicação de um questionário aos 32 docentes de três cursos de graduação em Nutrição de Instituições públicas e privadas de Belém-PA. Foi utilizado o software BioEstat versão 5.3, e aplicados os teste G e Qui-quadrado para verificar o grau de dependência entre as variáveis, com nível de significância de 0,05 . Resultados: Participaram 32 docentes, de ambos os sexos, com predominância do sexo feminino, com mais de 5 anos de atuação docente e titulação de mestre. 90,7\% dos docentes adotaram como estratégia de ensino o método tradicional de aulas expositivas com parcialidade do uso de metodologia ativa; apesar de afirmarem gostar de utilizar metodologias ativas $68,8 \%$ afirmaram adotar a forma metodológica mista considerando ser inovadora $(60 \%)$ e permitir a vivência pratica do conteúdo (96,9\%). 93,7\% dos docentes afirmaram ter conhecimento dos métodos ativos de ensino e serem motivados para diversificar a forma metodológica utilizada. Porém, sentem-se parcialmente preparados $(78,1 \%)$ para aplicar a metodologia ativa como método único de ensino. Conclusão: É necessário maior capacitação dos docentes para aplicação dos métodos ativos de ensino, através da busca do próprio profissional, também do alicerce das Instituições de Ensino Superior - IES.

Palavras-chave: Formação em saúde, Docência em nutrição, Práticas educacionais docentes.

\begin{abstract}
Objective: To evaluate the use of active methodologies as a teaching strategy in undergraduate courses in Nutrition of Belém-PA. Method: This is a cross-sectional, quantitative descriptive study with the application of a questionnaire to 32 professors from three undergraduate courses in Nutrition of public and private institutions in Belém-PA. The software BioEstat version 5.3 was used, and the $G$ and Chi-square tests were applied to verify the degree of dependence between the variables, with significance level of 0.05 . Results: A total of 32 female and male teachers, with more than 5 years of teaching experience and master's degree, participated. $90.7 \%$ of teachers adopted as a teaching strategy the traditional method of expository classes with partial use of active methodology; although they said they would like to use active methodologies, $68.8 \%$ stated that they adopt the mixed methodological method, considering that they are innovative $(60 \%)$ and that they can practice content (96.9\%). $93.7 \%$ of teachers stated that they were aware of the active teaching methods and were motivated to diversify the methodological form used. However, they feel partially prepared $(78.1 \%)$ to apply the active methodology as a single method of teaching. Conclusion: It is necessary to increase the capacity of teachers to apply the active methods of teaching, through the search of the professional, also the foundation of Higher education institutions - HEls.
\end{abstract}

Keywords: Health education, Teaching in nutrition, Teaching educational practices.

${ }^{1}$ Programa de Pós-graduação em Ensino em Saúde na Amazônia - ESA, Belém- PA.

*E-mail: crsfurtado@yahoo.com.br

2 Centro de Ensino Superior do Pará - CESUPA. Belém - PA.

3 Programa de Pós-graduação em Ensino em Saúde na Amazônia, Univ. do Estado do Pará, Belém - PA.

SUBMETIDO EM: 1/2019 


\section{RESUMEN}

Objetivo: Evaluar la utilización de las metodologías activas como estrategia de enseñanza en los cursos de graduación en Nutrición de Belém-PA. Método: El estudio es descriptivo transversal, cuantitativo con aplicación de un cuestionario a los 32 docentes de tres cursos de graduación en Nutrición de Instituciones públicas y privadas de Belém-PA. Se utilizó el software BioEstat versión 5.3, y se aplicaron las pruebas G y Qui-cuadrado para verificar el grado de dependencia entre las variables, con un nivel de significancia de 0,05. Resultados: Participaron 32 docentes, de ambos sexos, con predominancia del sexo femenino, con más de 5 años de actuación docente y titulación de maestro. El 90,7\% de los docentes adoptaron como estrategia de enseñanza el método tradicional de clases expositivas con parcialidad del uso de metodología activa; apesar de afirmar tener gusto de utilizar metodologías activas 68,8\% afirmaron adoptar la forma metodológica mixta considerando ser innovadora $(60 \%)$ y permitir la vivência práctica del contenido (96,9\%). El $93,7 \%$ de los docentes afirmaron tener conocimiento de los métodos activos de enseñanza y ser motivados para diversificar la forma metodológica utilizada. Sin embargo, se sienten parcialmente preparados $(78,1 \%)$ para aplicar la metodología activa como método único de enseñanza. Conclusión: Es necesaria una mayor capacitación de los docentes para la aplicación de los métodos activos de enseñanza, a través de la búsqueda del propio profesional, también del cimiento de lãs Instituciones de enseñanza superior - IES.

Palabras clave: Formación en salud, Docencia en nutrición, Prácticas educativas docentes.

\section{INTRODUÇÃO}

A literatura na área de educação em saúde revela a necessidade de mudanças no sistema educacional, o século XX trouxe esta necessidade de mudanças nos processos de produção do conhecimento, onde ainda prevalece o ensino tradicional com aulas expositivas, para que o foco agora seja no graduando, e para que as condutas profissionais sejam realizadas de forma mais consciente e segura (SEBOLD LF, et al., 2016).

A pedagogia hoje reconhece o sistema anterior como mera transmissão de informações sem que seja desenvolvida a criticidade do discente, e sem a preocupação com a aprendizagem significativa (FREIRE P, 2003). Desta forma o docente precisa compreender as mudanças pedagógicas e o novo papel que assume neste processo. $O$ discente assume, então, um papel mais ativo, onde ele é responsável pela construção de seu próprio conhecimento, exercendo atitudes críticas e construtivas (NETO JC, SOUSA FR, 2015).

As Metodologias Ativas (MA) surgem como estratégias facilitadoras do aprendizado significativo, mas cabe ao docente apoderar-se de competência, habilidades e atitudes para apresentar os métodos ativos de aprendizagem aos graduandos como proposta facilitadora de inserção do conhecimento, esta proposta só será verdadeiramente aceita quando o docente reconhecer seu novo papel neste processo, e o discente sentir-se motivado na busca do conhecimento. Na busca de novas formas de realizar o processo ensinoaprendizagem, quebrando as limitações do método tradicional e visualizando a amplitude para a construção do aprendizado significativo surgiram os métodos ativos de aprendizagem, entre outros destaca-se, o "Team Based Learning" (TBL), o "Problem Based Learning" (PBL), e a Problematização (SEBOLD LF, et al., 2016): O TBL- Aprendizagem Baseada em Equipes (ABE) ou do inglês Team Based Learning (TBL) é uma modalidade da educação problematizadora que permeia características do ensino tradicional com os benefícios do trabalho em pequenos grupos, provocando efetivamente a ruptura do paradigma do ensino centrado no professor, para o ensino centrado no aluno. A estratégia permite o trabalho em grupos de $10 \mathrm{e}$ até 25 alunos, dependendo da habilidade do professor. TBL Permite substituir ou complementar um curso nos modelos de aulas expositivas, pois admite seu desenvolvimento nas próprias salas de aula e a atuação de um docente por sala. Favorece o estudo prévio do graduando para o desenvolvimento das atividades em sala, o professor deve ser um especialista nos assuntos a serem desenvolvidos, porém não necessita do domínio do processo de trabalho em grupo. Os graduandos vão aprendendo o significado de trabalho colaborativo à medida que as unidades vão sendo desenvolvidas (CARRARO TE, et al., 2011; SEBOLT L, et al., 2010). O professor assume a função de facilitador para a aprendizagem em um ambiente de 
igualdade, utilizando as experiências e conhecimentos prévios dos graduandos como iscas para a busca da aprendizagem significativa; provoca mudanças na construção da aprendizagem, pela ampliação de conhecimentos e conteúdos, além do desenvolvimento do raciocínio crítico (BOLLELA VR, et al., 2014).

Quanto ao desenvolvimento do PBL - Aprendizagem baseada em problemas é realizada em pequenos grupos e um professor tutor para resolver diversos problemas e casos previamente pensados e preparados utilizando raciocínio lógico. Há existência de um Coordenador dentro do grupo a quem cabe a liderança e função de motivar a participação de toda a equipe; faz-se também necessário a existência de um secretário, que cuida da programação e organização do trabalho, o professor refere-se mais a dar sequência aos objetivos na discussão dos problemas. Essa metodologia baseia-se no ensino através da investigação por parte dos alunos e estímulos gerados a cada projeto, objetiva-se usar um problema para preparar este aluno ao invés de preparar o aluno para o problema (PERREIRA CF, et al., 2007). Consequentemente a aprendizagem toma como base a exploração do problema na tentativa de solução, identificação de deficiências, priorização do que é necessário aprofundar em questão do conteúdo, aplicação deste estudo no questionamento do problema, finalizando com a avaliação efetuada sobre os conteúdos aplicados. Professor não é mais o centro do processo de ensino. Possibilita a construção de novos conhecimentos e a aquisição de habilidades.

A metodologia da problematização apresenta como diferencial o uso de um problema real buscado pelos próprios alunos envolvidos. O grupo trabalha unido durante todo o processo e aplica conceitos teóricos para tratar a situação encontrada, os resultados aqui podem ser imprevisíveis visto que estão presentes variáveis externas reais (CARRARO TE, et al., 2011; SEBOLT LF, et al.,2010). Objetiva-se preparar o aluno para tomar decisões a partir de fatores surpresas e proporcionar o desenvolvimento da consciência, pois nem todos os projetos construídos em sala possibilitam a solução no contexto social encontrado. Necessita da existência do Tutor que deve planejar rigorosamente suas aulas e participar de maneira mais ativa, tempo disponível para a realização dos projetos, a disciplina específica em que eles serão realizados, e a integração de todo o corpo docente e discente no plano de aula. É o enfrentamento de uma situação nova, exigindo pensamento reflexivo, crítico e criativo a partir dos dados expressos na descrição do problema; identifica o problema e discute intervenção.

No alcance de seu objetivo, o presente estudo propôs-se avaliar a utilização de metodologias ativas como estratégia de ensino nos cursos de graduação em Nutrição de Belém-PA.

\section{MÉTODO}

Trata-se de um estudo descritivo transversal, quantitativo foi aplicado um formulário estruturado com perguntas fechadas aos docentes de três cursos de graduação em Nutrição do município de Belém - PA. A coleta de dados foi realizada no período entre abril e maio/2017, a partir da aprovação do Comitê de Ética em pesquisa (CAAE de no 65664117.0.0000.5174), e autorização das Coordenações das Instituições de Ensino Superior- IES coparticipantes, assim como assinatura do TCLE (Termo de Consentimento Livre e Esclarecido).

Objetivando coletar informações vivenciadas na prática profissional dos docentes e considerando a implantação ou não da nova estrutura curricular a partir do Projeto Pedagógico-PP de cada curso. O universo populacional foi de trinta e dois (32) docentes graduados em Nutrição, em condição de cargo efetivo do quadro funcional com mais de um ano de atividade docente, entre as instituições públicas (1) e privadas (2). O formulário foi dividido em três etapas: Identificação do docente; dados sobre a metodologia utilizada e dados sobre a capacitação docente.

O projeto foi apresentado ao corpo de docentes em reuniões ordinárias de colegiado para ciência dos mesmos. Foram utilizadas as seguintes estratégias de comunicação, seguindo Costa(2010): Inicialmente foi solicitado a secretaria de cada curso a identificação nominal e contatos dos docentes, para envio via email de convite, com esclarecimento dos os objetivos da pesquisa; do TCLE, e formulários de coleta de dados. Para as informações das respostas que não foram obtidas por meios eletrônicos, ou com devolução 
impressa, foi planejado um segundo re-envio de solicitação "on line" de preenchimento e devolução das informações, 4 a 8 dias após o envio do formulário inicial. Em seguida decorrida a segunda semana, os formulários não devolvidos e/ou não preenchidos, os dados foram coletados pela própria pesquisadora de forma presencial, na respectiva IES.

O tratamento estatístico dos dados foi realizado através do software BioEstat versão 5.3. Para caracterização dos dados quantitativos foram aplicados os testes $\mathrm{G}$ e Qui-quadrado para verificar o grau de dependência entre as variáveis associadas no estudo, considerando um nível de significância de 0,05.

\section{RESULTADOS E DISCUSSÃO}

Os dados aqui apresentam as características gerais de trinta e dois (32) docentes de ambos os sexos de três Instituições de Ensino Superior (IES) do curso de graduação em Nutrição do município de Belém-PA, onde houve predominância do gênero feminino com representação de $87 \%$ dos participantes; quanto ao tempo de atividade docente a maioria $(59,4 \%)$ afirmaram exercer atividade profissional por mais de cinco anos, e $87,5 \%$ dos docentes apresentaram titulação de Mestre (Tabela 1).

Tabela 1 - Características gerais dos docentes de três cursos de graduação em Nutrição do município de Belém-PA, 2017.

\begin{tabular}{lcc}
\hline CARACTERIZAÇÃO GERAL & N & $\%$ \\
\hline GÊNERO & & \\
Feminino & $\mathbf{2 8}$ & $\mathbf{8 7 . 0}$ \\
Masculino & 04 & 13.0 \\
$\quad$ Total & 32 & 100.0 \\
\hline TEMPO DE ATIVIDADE DOCENTE & & \\
1 - 05 anos & $\mathbf{1 3}$ & $\mathbf{4 0 . 6}$ \\
5 - 10 anos & 10 & 31.3 \\
> anos & 09 & 28.1 \\
$\quad$ Total & 32 & 100.0 \\
\hline TITULAÇ̃o & & \\
Especialização & 00 & 0.0 \\
Mestrado & $\mathbf{2 8}$ & $\mathbf{8 7 . 5}$ \\
Doutorado & 04 & 12.5 \\
Total & 32 & 100.0 \\
\hline
\end{tabular}

Fonte: Dados resultantes da pesquisa (2017).

Tais características foram semelhantes a encontrada em outros estudos na área da saúde, os quais constaram a predominância do sexo feminino e com titulação de mestre (BOSSINI ES, 2014; BISPO EP, et al, 2014). O número de docentes com doutorado é considerado pequeno, provavelmente pelo fato do estado do Pará ainda não possuir programas de mestrado e doutorado específicos na área da Nutrição, conforme verificado no portal de programas de pós-graduação da CAPES (Tabela 1).

Na Tabela 2 estão dispostos os resultados quanto a análise das estratégias de ensino utilizadas pelos docentes para o favorecimento do aprendizado dos alunos, observou-se que a estratégia metodológica mais adotada foi a do tipo Mista (tradicional com parcialidade de metodologias ativas).

Ao investigarmos a estratégia metodológica utilizada identificamos divergências nas respostas aos questionamentos: "qual a estratégia metodológica adotada" e "qual estratégia que mais gosta de usar" apesar de $68 \%$ dos docentes terem respondido que gostam mais de utilizar as metodologias ativas como estratégia de ensino, quando questionados sobre qual a estratégia metodológica realmente adotada, as aulas expositivas predominaram como estratégia de ensino utilizada durante o processo de Graduação, apesar de muitas vezes estarem associadas a metodologias ativas, o que denominavam como "parcialmente", e nesses casos não foi analisado o que realmente entende-se como parcialmente. Estudos de Guedes MJ et al. (2013); e Bossini ES (2014); afirmam que esta predominância de aulas expositivas, 
reflete que as práticas pedagógicas dos docentes estão voltadas para um modelo de ensino do tipo tradicional e que ainda necessita de maior rigor das IES em solicitar do docente a mudança no comportamento metodológico adotado. Porém a maioria das IES ao repensar em seu modelo de projeto pedagógico, já redesenharam um modelo de formação centrado no aluno como sujeito e agente de sua aprendizagem, tendo a figura do professor como facilitador, baseado em problemas cotidianos.

Dentre os resultados encontrados foi identificado que a estratégias de ensino que os docentes que mais gostam de utilizar são os métodos ativos referidos por $68,8 \%$, mesmo sendo utilizada a forma Mista (tradicional com parcialidade de metodologias ativas) por $90 \%$ do participante conforme apresentado na Tabela 2. Considerando assim esta forma mista, um método inovador por $60 \%$ dos docentes. Afirmaram também em $96,9 \%$ que está forma mista permite a vivência prática do conteúdo e não apenas o conhecimento teórico.

Tabela 2 - Percepção dos docentes em três cursos de graduação em Nutrição do município de Belém-PA, quanto a forma do método aplicado, 2017.

\begin{tabular}{lcc}
\hline ESTRATÉGIA METODOLÓGICA ADOTADA & N & $\%$ \\
\hline Apenas metodologia tradicional & 01 & 3.0 \\
Apenas metodologia ativa & 02 & 6.3 \\
Metodologia tradicional + Ativa (MISTA) & $\mathbf{9 0 . 7}$ \\
\hline MÉTODO QUE MAIS GOSTA DE UTILIZAR & $\mathbf{2 9}$ & \\
\hline Tradicional & & 28.1 \\
Metodologias ativas & 09 & $\mathbf{6 8 . 8}$ \\
Ambos & $\mathbf{2 2}$ & 3.1 \\
Total & 01 & 100.0 \\
\hline METODOLOGIA UTILIZADA É INOVADORA AO DISCENTE? & 32 & \\
\hline Sim & $\mathbf{1 9}$ & $\mathbf{6 0 . 0}$ \\
Não & 34.0 \\
Parcialmente & 6.0 \\
Total & 02 & 100.0 \\
\hline METODOLOGIA UTILIZADA PERMITE VIVÊNCIA PRÁTICA & 32 & \\
Sim & & \\
Não & $\mathbf{3 1}$ & $\mathbf{9 6 . 9}$ \\
Total & 01 & 3.1 \\
\hline
\end{tabular}

Fonte: Dados resultantes da pesquisa (2017).

O estudo afirma ainda existir nas IES a predominância do modelo tradicional de ensino, o qual o professor é o detentor do conhecimento e o aluno um mero receptor e reprodutor destes conhecimentos. Outros estudos afirmam que as estratégias de ensino utilizadas contribuem significativamente na absorção do conhecimento e precisam ser repensadas (VIEIRA OS, BAGGIO A e MARASCHIM R, 2007; SIGNORELLI MC, et al., 2010).

A literatura é extensa ao pesquisar a importância da utilização das, mas neste processo de mudança de paradigma no ensino, muitos destacam a necessidade que o docente tem de assumir uma nova postura para conduzir a aprendizagem significativa. Entretanto, ainda encontramos controvérsias nesta discussão, com critica na utilização do ensino tradicional, porém com afirmações que a manutenção de uma estrutura disciplinar conservadora mantendo a ordem e a disciplina não significa despreocupação com a formação (SOBRAL FR e CAMPOS CJ, 2012).

Ao questionarmos sobre a inovação do método de ensino, talvez os docentes possam estar equivocados ao responderem, pois a inovação não está na inserção de um novo método, mas na forma ou técnica de aplicá-lo tornando-o inovador. As metodologias precisam acompanhar os objetivos pretendidos se desejamos visualizar alunos construtores, alunos que vão em busca do conhecimento, precisamos utilizar metodologias que estimulem nossos alunos a exercerem estas práticas, de tomar decisões e avalia-las 
assim como a reflexão dos resultados que por ventura irão surgir a partir das ações por eles realizadas. Se desejamos alunos criativos, precisamos inseri-los em vários contextos os quais eles possam exercer suas habilidades de iniciativa e criatividade (ESCOBRAR SJ, 2013).

Ao questionar se as MA permitem vivência do conteúdo e não apenas conhecimento teórico, obteve resultado positivo em $75 \%$ dos pesquisados. Tais resultados mostram a preocupação dos docentes em utilizarem metodologias que favoreçam a vivência prática do conteúdo e não apenas a teoria. $A$ afirmação "vivenciar o conteúdo e não apenas o conhecimento teórico", leva ao alcance do principal objetivo, que é criar condições de qualificar o graduando para solucionar problemas reais do cotidiano da sociedade. $O$ foco parte não somente da escolha da estratégia, mas o importante é que as diferentes estratégias criem situações e abordem conteúdos que contribuam para a aprendizagem possibilitando ao discente uma transformação social (SOBRAL FR e CAMPOS CJ, 2012).

O conhecimento das estratégias de ensino e a sua aplicação deveria ser um requisito básico na formação de futuros docentes (ESCOBAR SJ, 2013). A caracterização da capacitação docente encontra-se descrita na Tabela 3.

Na Tabela 03 apresentamos os resultados da análise da capacitação docente, destes $93,7 \%$ afirmam conhecer os métodos ativos de ensino, $87,5 \%$ afirmam receber motivação pelas IES para a utilização dos métodos, e $68,7 \%$ confirmam ter recebido alguma forma de treinamento ou capacitação. Ao que se refere a classificação da capacitação recebida apenas $40,6 \%$ classificam como Bom, e 78,2\% dos docentes sentemse parcialmente preparados par utilizarem as metodologias ativas como único método de ensino.

Tabela 3 - Capacitação dos docentes de três cursos de graduação em Nutrição do município de Belém-PA, 2017.

\begin{tabular}{lcc}
\hline CAPACITAÇÃO DOCENTE & $\mathbf{N}$ & $\%$ \\
\hline CONHECIMENTO DE METODOLOGIAS ATIVAS & & \\
Sim & $\mathbf{3 0}$ & 93.7 \\
Não & 02 & 6.3 \\
Total & 32 & 100.0 \\
\hline MOTIVAÇÃO PELA IES PARA UTILIZAÇÃO DE METODOLOGIA ATIVA & & \\
Sim & $\mathbf{2 8}$ & $\mathbf{8 7 . 5}$ \\
Não & 04 & 12.5 \\
Total & 32 & 100.0 \\
\hline TREINAMENTO PARA UTILIZAÇÃO DE METODOLOGIA ATIVAS & \\
Sim & $\mathbf{2 2}$ & $\mathbf{6 8 . 7}$ \\
Não & 10 & 31.3 \\
Total & 32 & 100.0 \\
\hline CLASSIFICAÇÃO DA CAPACITAÇÃO & & \\
Bom & $\mathbf{1 3}$ & $\mathbf{4 0 . 6}$ \\
Regular & 09 & 28.1 \\
Ruim & 10 & 31.3 \\
Total & 32 & 100.0 \\
\hline SENTE-SE PREPARADO PARA USO DAS METODOLOGIAS ATIVAS & & 15.6 \\
Sim & 05 & 6.3 \\
Não & 02 & 78.1 \\
Parcialmente & $\mathbf{2 5}$ \\
Total & 32 & 100.0 \\
\hline
\end{tabular}

Fonte: Dados resultantes da pesquisa (2017).

Quanto a caracterização da capacitação docente pode-se descrever que a presente pesquisa demonstra que quase a totalidade (93.8\%) dos docentes afirmaram ter conhecimento ou já ter tido algum contato com um método ativo de ensino (Tabela 3). A Tabela 2, mostra que 90,7\% destes docentes referem de alguma forma utilizar MA no decorrer do conteúdo apresentado. 
Tais resultados não eximem sob qualquer hipótese o compromisso de o docente ir em busca deste conhecimento pois as novas Diretrizes Curriculares Nacional-DCNs vêm nos despertar para a real necessidade da mudança de métodos tradicionalmente utilizados, de forma a tornar o ensino centrado no aluno e o docente o facilitador do processo. E os novos modelos de projetos pedagógicos dos cursos de graduação da área da saúde devem ser criados e/ou remodelados com base no novo perfil profissional que almejamos. O docente necessita obter domínio de métodos ativos que o permita sair do limite de quatro paredes e atuar na inserção teoria/prática, utilizando a educação em serviço, fazendo-se dispensável a sala de aula e o projetor (BRASIL, 1996). No presente estudo ao ser lançado o questionamento aos docentes pesquisados com vista a saber sobre a existência de motivação pela IES para utilizar a forma metodológica ativa, os resultados comprovam o pouco investimento da diversificação dos métodos utilizados. Os resultados também mostram que mesmo a maioria (87,5\%) tendo informado receber motivação e treinamento pelas IES para utilizar metodologias ativas de ensino, ainda 90,7\% dos docentes afirmam utilizar o método tradicional, mesmo que com alguma parcialidade de métodos ativos (não foi pesquisado sobre o entendimento de parcialidade).

No presente estudo, $69 \%$ dos docentes entrevistados afirmam ter recebido alguma forma de capacitação para utilização de MA, destes $40 \%$ consideraram a capacitação recebida como conceito bom. Ao realizar uma capacitação deve-se analisar conteúdo, carga horária, horário de realização, metodologia aplicada, recursos necessários, forma de avaliação e aplicação do conhecimento, assim como a formulação do problema a ser apresentado, e a análise da obtenção dos resultados esperados (CAVALLI SB e SALAY E, 2007), tais fatores podem justificar o conceito atribuído.

Com vistas aos resultados do presente estudo torna-se transparente a necessidade de maior atenção quanto a capacitação docente para aplicação dos métodos ativos de ensino, necessitando além da busca individual do profissional, mas, também de subsídios das IES quanto ao desenvolvimento de habilidades e aplicação de métodos facilitadores da aprendizagem.

Rocha EF (2014); após realizar sua pesquisa sobre as "Metodologias Ativas: um desafio além das quatro paredes da sala de aula", afirma que ainda é comum a reação de alguns professores que desistem de utilizar a metodologia ativa porque está preso a alguns obstáculos como a crença que as metodologias ativas estão ligadas a tecnologia, ou ser um método de difícil desenvolvimento e que requer muito tempo para a aplicação ou ainda não conseguir inseri-lo em seu conteúdo. Fatos que ainda demonstram a insegurança do docente quanto ao domínio e aplicação dos métodos, visto que foram formados pela metodologia tradicional, e que ainda não receberam capacitação significativa ou ainda de alguma forma não sentem a necessidade de mudança.

Assim a proposta inovadora ainda precisa ser pauta de muitos debates, considerando a atual cultura do aluno passivo e o processo deficiente de formação dos professores, principais construtores da mudança no processo de aprendizagem (ROCHA EF, 2014); assim como o aluno não está preparado para aprender como protagonista, além da necessidade do conhecimento prévio; hábito de leitura, administração de tempo, espírito de cooperação e relações interpessoais, perfis que ainda são necessários desenvolver na comunidade acadêmica. Mas o professor precisa se permitir aprender a mudar, sair da proteção da justificativa de ter sido formado na metodologia tradicional e tornar-se a principal ferramenta desta mudança e, assim contribuir com o crescimento do aluno (MORAM JM, 2014).

Bispo EP et al. (2014); que estudaram a "Interdisciplinaridade no ensino em saúde" descrevem em seus resultados que a maioria dos docentes, não teve uma formação específica para atuar em suas áreas de conhecimento. Assim, esta observação pode nos ajudar a justificar por que os profissionais não se sentem totalmente preparados para esta prática profissional específica, o que pode retratar uma deficiência na formação acadêmica pelos docentes. Esta realidade ajuda a compreender os resultados dessa pesquisa, corroborando também com os resultados obtidos por Escobar SJ (2013), o qual afirma que os cursos de bacharelado não exigem no decorrer de seus projetos a qualificação pedagógica, permitindo a abertura de uma lacuna aos profissionais que irão atuar na educação. 
O empoderamento do conhecimento de MA e o novo papel do professor só ocorrerá quando o docente almejar a mudança pedagógica em busca do novo perfil do discente, e assim assumir seu verdadeiro compromisso do aprender a ensinar com base no aprendizado significativo (BULGRAEN VV, 2010).

A preocupação de Costa MV (2010); assemelha-se a de Bulgraen VV (2010); e é compartilhada também por Vieira JL, Machado VM e Vieira JE (2011); e Escobar SJ (2013) ao destacar que a pouca habilidade dos docente na aplicação de métodos ativos está relacionada a formação do docente, não apenas nas graduações de Nutrição mas a falta de formação dos docentes em toda área da saúde, o que torna a formação de muitos futuros profissionais deficiente. Vistas a isso os cursos de pós-graduação estão utilizando a capacitação didática pedagógica como matéria obrigatória em seus cursos.

\section{CONCLUSÃO}

Entre os docentes que participaram do estudo, nenhum utilizava as metodologias ativas como único método de ensino. A maioria preferiu utilizar o método tradicional com aulas expositivas e uso parcial de metodologia ativa em algumas abordagens, mesmo afirmando gostarem do novo método, utilizaram um misto de técnicas de ensino (tradicional + ativa), afirmaram também receber motivação e capacitação das IES para utilizar metodologias ativas. Porém a maioria ainda sentem-se parcialmente preparados para o uso de métodos ativos de ensino apresentando-se claro a necessidade de maior atenção quanto à qualificação dos mesmos para aplicação desses métodos em suas aulas, necessitando além da busca própria de cada profissional, a contribuição das IES quanto ao desenvolvimento de habilidades por parte do docente e a aplicação dos métodos ativos facilitadores da aprendizagem. Destacam-se os esforços por parte dos docentes que mesmo afirmando ainda sentirem-se parcialmente preparados para a aplicação dos métodos ativos como único método de ensino, buscam de alguma forma introduzir a MA. Porém, no presente estudo identificou-se também que o professor ainda não assumiu seu novo papel, de mediador da aprendizagem, pois o processo de formação ainda é baseado no ensino tradicional, transmissivo, centrado no professor, e baixa utilização de metodologias que tornem o ensino significante e motivador. Conclui-se que no município de Belém-PA, os cursos de graduação em Nutrição que participaram do estudo necessitam adaptar-se ao processo de mudanças para atender ao novo perfil profissional com competências, habilidades e atitudes requeridas pelas DCN. Há necessidade de implantação de um programa de capacitação continuada aos docentes, constituído por oficinas com programação definida conforme as necessidades identificadas.

\section{REFERÊNCIAS}

1. BRASIL, Ministério da Educação. Conselho Nacional de Educação.Lei no 9.394, de 20 de dezembro de 1996. Estabelece as diretrizes e bases da educação nacional. Brasília: Planalto. 1996.

2. BISPO EP, et al. Interdisciplinaridade no ensino em saúde: o olhar do preceptor na saúde da família. Interface, 2014; Botucatu, v. 18, n. 49, p. 337-350, jun.

3. BOLLELA VR. et al. Aprendizagem baseada em equipes: da teoria à prática. Revista de Medicina, 2014; Ribeirão Preto. 47(3), p. 293-300.

4. BOSSINI ES. Análise das estratégias de ensino e da aprendizagem no processo de formação do fisioterapeuta. Dissertação (Mestrado em Ensino em Saúde na Amazônia) - Universidade do Estado do Pará. Belém. 2014, 140p.

5. BULGRAEN VV. Revista Conteúdo, Capivari, v.1, n.4, ago./dez. 2010 - ISSN 1807-9539

6. CARRARO TE, et al. Socialização como processo dinâmico de aprendizagem na enfermagem. Uma proposta na metodologia ativa. Invest. Educ.Enferm,2011; Medelim/Colômbia. v. 29 (2).

7. CAVALLI SB, SALAY E. Gestão de pessoas em unidade produtora de refeições comerciais e a segurança alimentar. Revista de Nutrição, 2007; Vol 20 n 6 Campinas. Nov/ dez.

8. COSTA MV. Sobre as contribuições das análises culturais para a formação dos professores do início do século XXI. Educar, 2010; Curitiba, n.37, p.129-152, maio/agosto. Editora: UFPR.

9. ESCOBAR SJM. Percepção de alunas do curso de nutrição acerca da prática pedagógica de seus professores. Revista P@rtes 2013; São Paulo, v. 15, p. 01.

10. FREIRE P. Educação e mudança. 27 ed. Rio de Janeiro: Paz e Terra, 2003. 
11. GUEDES MJP, et al. Ensino e práticas da fisioterapia aplicada à criança na formação do fisioterapeuta. Fisioterapia e movimento, 2013; vol.26, n.2, pp.291-305.

12. MORAN JM. A educação que desejamos: novos desafios e como chegar lá. 5. ed. Campinas: Papirus, 2014.

13. NETO JCS, SOUSA FR. O papel do professor na formação de sujeitos: obstáculos e desafios de uma educação transformadora. II Congresso Nacional de Educação - CONEDU. Campina Grande, Paraíba. 2015.

14. PEREIRA CF, et al. Aprendizagem Baseada em Problemas (ABP): Uma proposta inovadora para os cursos de engenharia. Anais XIV UnicenP, 2007; Simpósio de Engenharia de Produção, Curitiba.

15. ROCHA EF. Metodologias ativas: um desafio além das quatro paredes da sala de aula. Associação Brasileira de Educação à Distância - ABED. 2014.

16. SEBOLD LF, et al. Metodologias ativas: uma inovação na disciplina de fundamentos para o cuidado profissional de enfermagem. Cogitare Enfermagem, 2010; out/dez.

17. SIGNORELLI MC, et al. Um projeto político pedagógico de graduação em fisioterapia pautado em três eixos curriculares. Fisioterapia e movimento. 2010, vol.23, n.2, pp.331-340. ISSN 1980-5918.

18. SOBRAL FR, CAMPOS CJG. Utilização de metodologia ativa no ensino e assistência de enfermagem na produção nacional: revisão integrativa. Revista Esc Enferm USP, 2012; São Paulo, vol.46, n.1, p.208-218.

19. VIEIRA PS, et al. Profissionais da área da saúde: a busca pela capacitação em educação especial. Saúde em Revista, 2007; v. 9, n. 21, p. 41-48.

20. VIEIRA JLM, et al. Formação e Seleção de Docentes Para Currículos Inovadores Na Graduação Em Saúde. Revista Brasileira De Educação Médica, 2011; Rio de Janeiro, v. 35, n. 3, p. 326-333, Sept. 